\title{
Pain Hypervigilance Influences Cortical Processing and Habituation to Painful Stimuli in Healthy Subjects: A Cross Sectional Pain ERP Study
}

\author{
Catherine J Vossen ${ }^{1 *}$, Rosan Luijcks ${ }^{2}$, Elbert A Joosten ${ }^{1}$, Jim van 0s ${ }^{2,3,4}$ and Richel Lousberg ${ }^{2}$ \\ ${ }^{1}$ Department of Anesthesiology and Pain Medicine, Maastricht University Medical Centre, Netherlands \\ ${ }^{2}$ Department of Psychiatry \& Psychology, Maastricht University Medical Centre, Netherlands
}

${ }^{3}$ Department of Psychosis Studies, Institute of Psychiatry \& King's College London, United Kingdom

${ }^{4}$ Department of Psychiatry, University Medical Centre Utrecht, Netherlands

Submission: February 15, 2019; Published: February 25, 2019

*Corresponding author: CJ Vossen, Department of Anesthesiology and Pain Medicine, Maastricht University Medical Centre, P.0. Box 5800, 6202 AZ Maastricht, Netherlands

\begin{abstract}
Pain hypervigilance can be detected at trait level in pain-free individuals and may represent a predisposing factor for chronic pain. Habituation to pain is impaired in several chronic pain populations. This experimental study investigated the influence of pain hypervigilance on cortical processing and habituation to painful stimuli. Forty-six pain-free participants underwent a habituation pain rating protocol of 25 painful somatosensory electrical stimuli, while recording EEG. Pain hypervigilance was assessed with the Pain Vigilance Awareness Questionnaire (PVAQ). At single trial level, the whole one-second post stimulus epoch was partitioned in 20-ms Event-Related Fixed-Interval Areas (ERFIAs). ERFIAs were used as dependent variables in multilevel models in which the PVAQ score was the variable of primary interest. PVAQ interaction effects with trial number were modeled to assess the influence of PVAQ on habituation. Forty-two subjects were eligible for analysis. Significant main effects for PVAQ were seen from 440-580ms, meaning that high PVAQ scores were associated with more positive single trial areas for that region, compared to low scores. The habituation course over 25 stimuli differed significantly between the two PVAQ groups from 480 $600 \mathrm{~ms}$. Pain hypervigilance impacts cortical processing of painful stimuli, suggesting that pain hypervigilance may modulate the pain experience through altered cortical habituation.
\end{abstract}

Keywords: Pain Hypervigilance; Event-Related Fixed-Interval Areas (ERFIA); Habituation; Pain; EEG

Perspective: This study shows that pain hypervigilance impacts cortical processing of pain and habituation to painful stimuli, suggesting that pain hypervigilance may modulate the pain experience. Insight in psychological mechanisms influencing pain processing and habituation could help the clinician identify psychological vulnerability to pain and may contribute to the management of pain.

Abbreviations: ERFIA: Event Related Fixed Interval Areas; ERP: Event Related Potential; ISI: Interstimulus interval; PVAQ: Pain Vigilance Awareness Questionnaire

\section{Introduction}

Hypervigilance is defined as the constant scanning of the body for somatic and pain sensation [1]. An excessive attention specifically to pain sensations is termed pain hypervigilance [2]. Pain hypervigilance may be one of the mechanisms through which pain-related fear can result in reports of higher pain intensity [3-6]. Pain hypervigilance can be assessed by the Pain Vigilance Awareness Questionnaire (PVAQ) [3]. This questionnaire was originally developed to assess attention to pain in chronic pain patients and was later also validated for non-clinical and pain-free study populations $[7,8]$.
Hypervigilance can already be apparent in pain-free individuals and as such may be a predisposing factor in the onset of chronic pain disorders [9-11]. Rolmann postulated that hypervigilant individuals may be biologically predisposed, and that hypervigilance may serve as a risk factor for the development of chronic pain syndromes [9-12]. Pain hypervigilance is thought to be associated with central sensitization, a mechanism in the nociceptive system, leading to increased clinical and experimental pain $[13,14]$. However, a pain experience not only results from nociceptive but also from antinociceptive mechanisms [15]. One of the most fundamental forms of learning is habituation. Habituation is 
defined as a physiological decrease in response to repetitive sensory stimulation $[16,17]$. In pain research, habituation is a process that is thought to function as a central antinociceptive mechanism $[18,19]$. Consequently, an impaired habituation to pain imbalances nociceptive and antinociceptive mechanisms and may result in a more severe pain experience. Both mechanisms are influenced by cognitive processes, such as fear and hypervigilance $[5,20]$.

In the context of the current investigation, two questions were addressed:

a) Since hypervigilance may represent a risk factor for the development of chronic pain, do hypervigilant pain-free individuals already display an altered cortical processing to painful stimuli compared to non-hypervigilant pain-free individuals?

b) Does pain hypervigilance moderate antinociceptive mechanisms through a decrease in habituation to painful stimuli?

One of the tools to investigate cortical processing of pain and habituation are Event-Related Potentials (ERPs) [21]. Pain ERPs are time-locked responses to somatosensory painful stimuli, derived from a continuous electroencephalogram (EEG) [22]. Specific deflections in the pain-ERP, such as the N2 and N2-P2 peakto-peak amplitude in the pain ERP are thought to be related to stimulus characteristics, such as intensity [22-25], and processes, such as attention [26,27] and habituation [28-30]. Recently, a new technique, called the Event-Related Fixed-Interval Area (ERFIA) multilevel technique, was developed for the analysis of ERPs [31]. This method enables the study of the whole post-stimulus epoch of an ERP and the habituation of repeated stimuli in a flexible way. For example, to study habituation, a linear function for stimulus number can be incorporated in the model to investigate a linear decline of 20-ms areas at a fixed post stimulus interval (i.e. ERFIAs), over the course of 25 stimuli. Consequently, by modeling a parabolic (quadratic) function, an initial decrease and later increase in response over the course of 25 stimuli can be investigated, representing an impaired habituation. In this article we will use the term linear habituation for the linear function and dishabituation for the quadratic function. In addition, this type of modeling creates the possibility to investigate the impact of psychological variables in relation to habituation on the whole epoch.

The aim of the present study was to investigate the two abovementioned issues in a habituation protocol of 25 somatosensory electrical stimuli:

a) Does a relationship exist between pain hypervigilance in pain-free individuals and event-related fixed interval areas after painful stimuli? Since a higher PVAQ score is associated with a higher pain intensity report, it was hypothesized that a high PVAQ score would be associated with a more negative N2 region and a more positive $\mathrm{P} 2$ region;

b) Does pain hypervigilance influence the habituation course of painful stimuli? It was hypothesized that a higher PVAQ score would be associated with a decreased linear habituation or more pronounced quadratic dishabituation. No a priori assumption concerning the latency range could be made, since no previous results exist. As secondary outcome, pain report, measured with NRS, was investigated.

\section{Methods}

\section{Participants}

The study was approved by the Medical Ethics Committee of the Maastricht University Medical Centre (NL40284.068.12/ METC 12-3-015). Participants consisted of a sample of the general population of Maastricht and were recruited using flyers. The flyers were delivered in 5 different neighborhoods in Maastricht. Inclusion criteria were an age between 18 and 65 years and a good understanding of the Dutch language. Exclusion criteria were:

a) Structural use of psychoactive medications such as antipsychotics, antidepressants, antiepileptics and/or anxiolytics during the past year,

b) Regular use of alcohol $>10 \mathrm{u}$ /day during the past year,

c) Epilepsy,

d) Psychotic disorder,

e) A visual or hearing disability,

f) Analphabetism or dyslexia. Subjects were asked not to take alcoholic beverages the evening prior to the experiment and to refrain from caffeine-containing beverages three hours before the start of the experiment.

Before the experiment, written informed consent was obtained. Compensation for the period of the whole experiment was 50 euros. Of a total of 111 subjects, participating in the original study, 46 subjects were pain free. The selection was made based on the following criteria: (1) no pain complaints at the moment of the experiment (BPI), nor during the six months before the experiment (SF-36). In addition, participants did not use pain medication. Since the present study was part of a larger dataset no a priori study size calculation was made.

\section{Questionnaires}

Before the EEG measurement, subjects were asked to complete the following three questionnaires:

a) Short-Form Health Survey (SF-36), consisting of 36 items, in order to evaluate the general health status and in particular the subscale bodily pain, [32]

b) Brief Pain Inventory Short Form (BPI-SF), which consists of 9 items, exploring pain complaints, [33,34]

c) The Pain Vigilance Awareness Questionnaire (PVAQ) which has 16 items and considers the pain behavior with respect to attention to pain and attention to changes in pain 
[8]. In addition to these questionnaires, questions regarding age, sex, pain complaints in the last six months, nature of pain complaints and medication use were also included.

\section{Electro-shocker and stimuli}

An electro-shocker (type Shocko-100-AA-20, developed by Maastricht Instruments BV and approved for use in experimental studies) was used to deliver electroshocks. Electrical pulse stimuli (duration 10 milliseconds) were applied intracutaneously on the left middle finger, as per Bromm and Meier [35]. Using this method, a small lumen in the epidermis was prepared with a dental gimlet. Care was taken that the procedure was not painful. In the prepared lumen, a golden electrode was placed and fixed with tape. A grounding wrist strap (3Mtm wrist strap, WBB-AFWS61M) was attached to the wrist proximally to the prepared finger. In order to determine the intensity of the stimuli for the habituation protocol, the sensation and pain thresholds were measured. The sensation and pain thresholds were determined by gradually increasing the intensity of the stimulus, starting at zero intensity. The first intensity that was consciously experienced was defined as the sensation threshold; the first intensity that was experienced as painful was defined as the pain threshold. This procedure was repeated 3 times to generate a reliable measurement.

\section{Habituation protocol}

Based on the subject's difference between the sensation and pain thresholds, a stimulus $25 \%$ above pain threshold was calculated as follows: Delivered habituation stimulus = pain threshold $+0.25^{*}$ (pain threshold - sensation threshold). This intensity level was experienced as painful, nonetheless still acceptable. The habituation protocol consisted of 25 identical stimuli, with durations of 10 milliseconds. Inter-stimulus intervals (ISIs) ranged between 9 and 11 seconds. Subjects were instructed that they would undergo a series of stimuli and that the task was to determine differences between the stimuli. The intensity of the stimuli was unknown to the subject, as was the number of stimuli that would be administered. Subjects were asked to rate the intensity of each stimulus on a scale from 0 (no sensation) to 100 (the most excruciating pain imaginable). For standardization purposes, subjects were asked to rate the first stimulus as 60 . In addition, subjects were instructed to wait for two seconds after each stimulus before rating the intensity of the stimulus

\section{EEG measurement}

The EEG recordings took place in an electrically and sound-shielded cubicle $(7.1 \mathrm{~m} 2)$. For the EEG recording, the Brain Amp Amplifier and Brain Vision Software were used. EEG recordings were sampled at $1000 \mathrm{~Hz}$. $\mathrm{Ag} / \mathrm{AgCl}$ electrodes were placed on respectively Fz, F3, F4, Cz, C3, C4, T3, T4, Pz, P3, P4, Oz, 01 and 02, using the international 10-20 system [36]. Reference electrodes were placed on the earlobes. A ground electrode was fixed at Fpz. To measure vertical eye movements, Electro-Oculogram (EOG) electrodes were placed 1 centimeter under the midline of the right and left eye. All electrodes were fixed using conductive paste.

\section{Data processing}

With Analyzer 2.0 (Brain Products, München, Germany) software, trials were segmented from the continuous EEG, from $200 \mathrm{~ms}$ before the stimulus to $1000 \mathrm{~ms}$ post stimulus. Data were offline band-pass filtered $(0-50 \mathrm{~Hz})$ and baseline-corrected (interval $-200 \mathrm{~ms}$ to $0 \mathrm{~ms}$ ). For each subject, the data (microvolts) for each millisecond (from -200 to $1000 \mathrm{~ms}$ ) of all electrodes and EOG channels were imported into SPSS 21.0. Subsequently, a multilevel dataset was built for each subject, using a syntax file in which the following calculations were made: (1) twenty-millisecond event-related fixed-interval areas (ERFIAs) were calculated from 0 to $1000 \mathrm{~ms}$ post stimulus, partitioning the whole epoch in 50 consecutive 20-ms areas (ERFIAs) per trial per EEG electrode per subject (2) Additionally, maximum and minimum values of the EOG channel were selected per 20-ms ERFIA. (3) Questionnaire data were added to the dataset. Next, all cases were added in order to obtain a full multilevel dataset. EOG left and right activity were included per 20-ms ERFIA in the analysis as covariates.

\section{Statistical analysis}

Multilevel regression analyses were carried out separately for each EEG electrode and for every 20-ms ERFIA period. The 20ms ERFIAs were used as the dependent variable. The dependent variables were assessed for normality. Subjects represented the highest level in the model and trial number (1-25 stimuli) was the repeated measure within subject. We made the assumption that subjects differ from one another with regard to habituation. Consequently, random effects, such as a random intercept and a random slope for trial number were included. An AR-1 covariance structure was used, assuming that trials next to each other are more correlated compared to trials further apart. As in previous studies, linear habituation (trial number) and dishabituation (parabolic relationship, computed as trial*trial) were modelled $[31,37]$. A linear functions with a negative coefficient represents a linear decline in ERFIAs over the course of 25 stimuli. A quadratic function, in which the parabola opens upward represents initial habituation after which a sensitization process (or dishabituation) occurs. For the research question, the main effect of PVAQ on the post-stimulus EEG was investigated, using the following basic model: ERFIAs of a specific 20-ms range and electrode (ERFIA s20-ms $_{\text {s }}$ range' location) served as the dependent variable, which was modelled as a function of the following fixed factors: trial number, $\operatorname{tri}_{\text {al- }}$ quadratic' $\mathrm{PVAQ}_{\text {median split' }}$ age, sex, pain threshold, sensation threshold, EOG left and EOG right. For the second research question, the investigation of the influence of pain hypervigilance on habituation and dishabituation, two two-way interactions were incorporated in the model, namely $\mathrm{PVAQ}_{\text {median split }}{ }^{*}$ habituation ${ }_{\text {linear }}$ and $\mathrm{PVAQ}_{\text {median }}$ split* $^{*}$ habituation $_{\text {quadratic. }}$

The analyses were performed separately for each 20-ms ERFIA $(0-1000 \mathrm{~ms}$ post stimulus) for all 14 cranial locations. For this large number of statistical tests, a correction for multiple testing should be performed. However, the analyses are explorative in nature. Therefore, we chose not to define a specific P-value for 
statistical significance. Instead, we considered only robust effects (3 or more consecutive 20-ms ERFIAs) with P-values $\leq .05$ as significant. For low-power interaction effects, we considered robust effects with P-values $\leq .10$ as significant.

The results were summarized in so-called ERFIA predictor blots, in which the columns represent 50 consecutive 20-ms ERFIAs, and the rows represent the EEG electrodes of a given predictor. In each row, cells were given a color when T-values were <-2 or $>2$. A red color indicated a positive significant T-value, a blue

Results

\section{Subject characteristics}

Table 1: Characteristics of the study participants.

\begin{tabular}{|c|c|c|c|c|}
\hline & Total & PVAQ Low & PVAQ High & P-values \\
\hline $\mathrm{N}$ & 42 & 21 & 21 & \\
\hline Mean PVAQ score (sd) & $28(13.6)$ & $16.5(6.7)$ & $39.5(7.5)$ & $\mathrm{P}<0.0001$ \\
\hline Mean age, years (sd) & $35.8(16.9)$ & $35.4(17.8)$ & $36.3(16.5)$ & ns \\
\hline Sex M/F & $19 / 23$ & $8 / 13$ & $11 / 10$ & ns \\
\hline Sensation threshold, $\mathrm{mA}$ & $0.34 \mathrm{~mA}$ & $0.32(0.18)$ & $0.35(0.15)$ & ns \\
\hline Pain threshold, mA & $1.13 \mathrm{~mA}$ & $1.1(0.68)$ & $1.2(0.63)$ & ns \\
\hline NRS 0-100 (sd) & $57(9.8)$ & $57(8.7)$ & $56(11.1)$ & ns \\
\hline
\end{tabular}

PVAQ: Pain Vigilance Awareness Questionnaire; sd: standard deviation; ns: non-significant; NRS: Numerical Rating Score

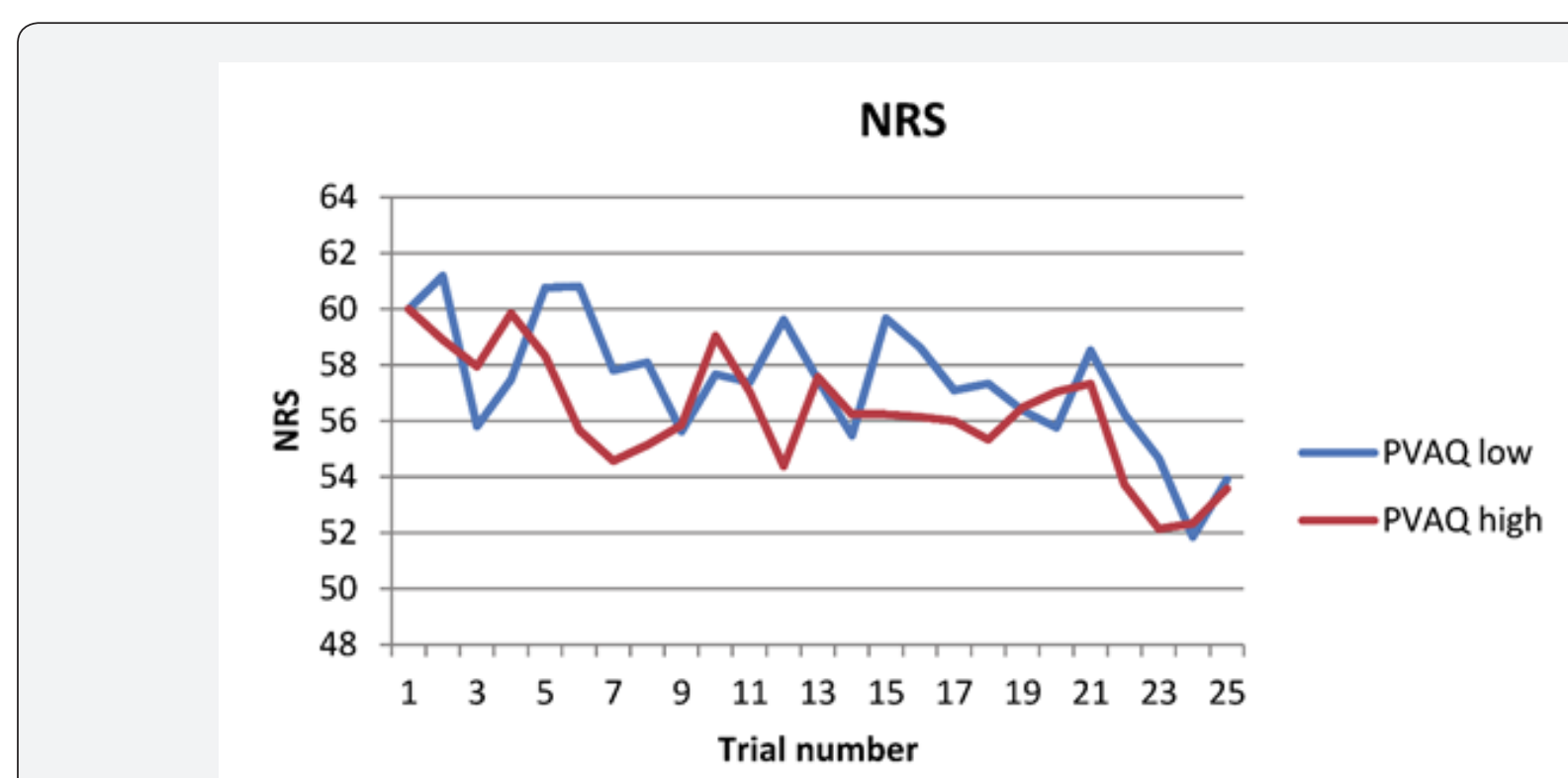

Figure 1: Pain intensity (NRS) of the 25 trials for PVAQ high and PVAQ low.

A total of 111 subjects were enrolled in the study. The inclusion took place from April 2012 until August 2014. Of a total of 111 participants, 46 subjects were pain free. Of these 46 subjects, 42 were eligible for analysis, one subject was excluded because of inadequate EEG measurement and three subjects had missing data on the PVAQ questionnaire. The PVAQ was dichotomized around the median split of a score of 29 . In Table 1 , the characteristics of the analyzable subjects are summarized. The group with a high PVAQ score did not significantly differ from the low PVAQ color a significant negative T-value. All statistical analyses were performed with SPSS 21.0. Also, the influence of pain hypervigilance on the secondary outcome of pain report, measured with NRS, was investigated, as was time course. NRS was the dependent variable and modelled as a function of the following variables: trial number, trialquadratic, PVAQmedian split, age, sex, pain threshold, sensation threshold, trial number* $\mathrm{PVAQ}_{\text {median split }}$ and tri alquadratic* $^{*} P V A Q_{\text {median split }}$ An overall course of NRS across the 25 stimuli was constructed and compared between the two PVAQ groups. 
wise, there were no different time courses of the NRS scores for the groups. There was no difference in NRS between the groups, nor did NRS course significantly differ between the groups.

\section{Main effects of pain hypervigilance and habituation on the event-related EEG}

Significant positive main associations between PVAQ and event-related EEG were mainly seen in the region from 440 to 580ms, except for F3, F4, C4 and T3 (Figure 2). This indicates that high PVAQ scores were associated with larger areas for that region, compared to low scores. In Figure 3A, a grand average ERP is depicted for participants with a high and a low PVAQ score at Cz. In the region of 460-560ms, an overall statistically significant difference for the PVAQ groups was observed for Cz (Figure 3A). With respect to habituation, for trial number (linear habituation) and trialquadratic (quadratic habituation), significant main effects were observed in a broad range of 140 to $460 \mathrm{~ms}$ for all electrodes. For the electrodes Fz, F3 and F4, main habituation effects were also seen in the area from 620 to $640 \mathrm{~ms}$ (Figure 2B \& 2C). In addition, significant main effects for sensory and pain thresholds after $400 \mathrm{~ms}$ were apparent across almost all electrodes (Figure 2D \& 2E).
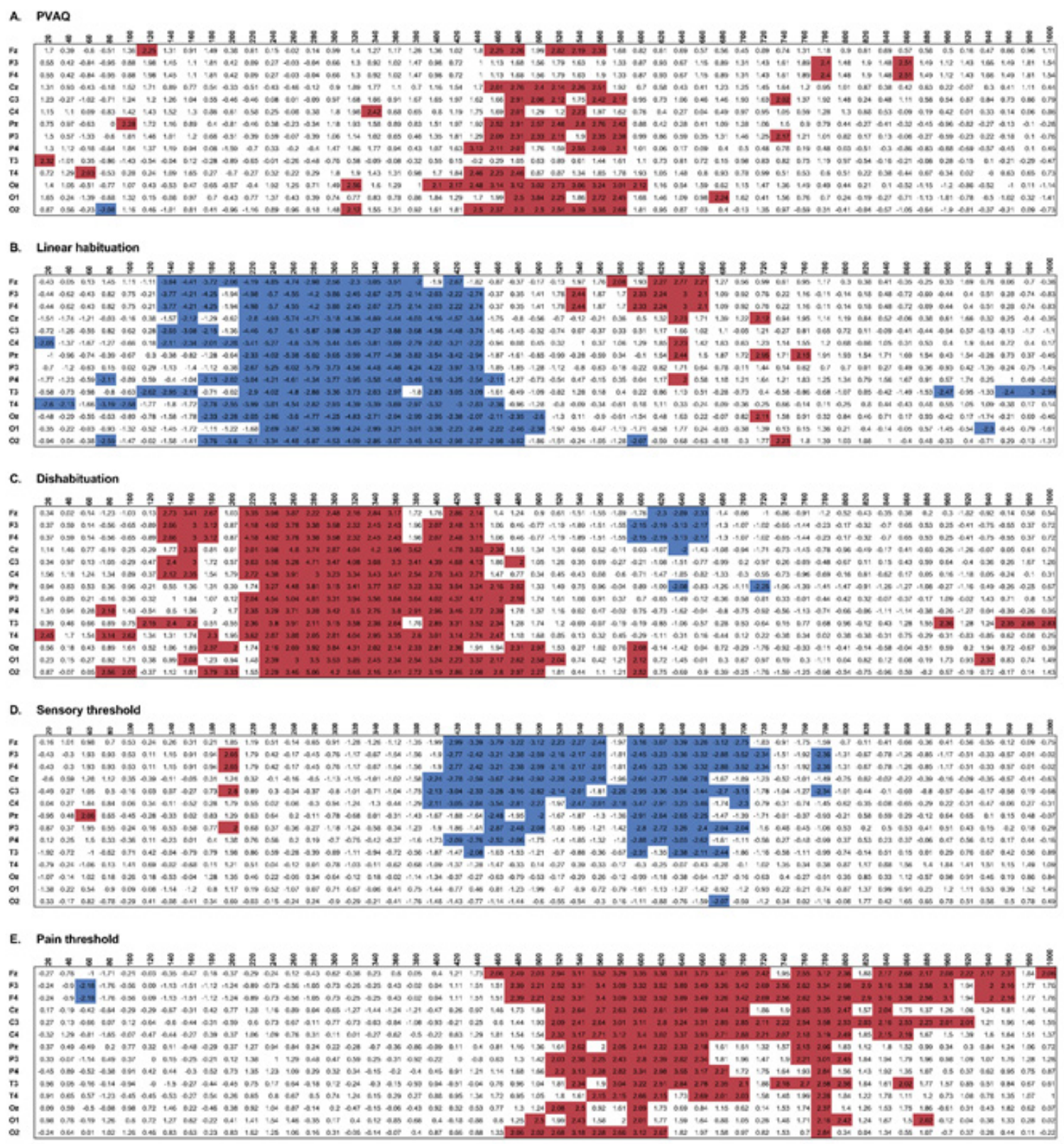

Figure 2D-2E: Pain intensity (NRS) of the 25 trials for PVAQ high and PVAQ low.

20-ms ERFIAs whereas rows display cranial locations. Cells with significant results are colored $(p<.05)$ and the plus or minus sign expresses the direction of the relationship. 


\section{Pain hypervigilance in relation to (dis)habituation}

To study the influence of pain hypervigilance on habituation, interaction effects of PVAQ with linear habituation and quadratic habituation (dishabituation) were added to the model. Interaction effects of pain hypervigilance with linear habituation and quadratic trial number effect were seen especially in the latency range from 480 to $600 \mathrm{~ms}$ (Figure 4). A positive coefficient is indicative for dishabituation, representing a parabola which opens upward. For the electrodes F4 and T4, significant effects were observed in a larger latency range. Significant robust effects were seen for F4, Cz, Pz, P3, P4 and T4. No effects were found for Fz, F3, $\mathrm{T} 3, \mathrm{Oz}$ and $\mathrm{O} 1$ (Figure 4). With respect to the results of random effects, the intercept was significant in almost all models, whereas the random slope for trial number was significant in approximately $50 \%$ of the models.

\section{Post-hoc analyses}

In order to test the robustness of the interaction effects found in the region from 480 to $600 \mathrm{~ms}$, we performed some post-hoc analyses. The sum of the ERFIAs 480 to $600 \mathrm{~ms}$ was calculated at trial level and was used as dependent variable for the interaction model. Six of the 14 electrodes showed significant PVAQ*trial interaction effects, namely F4, Cz, Pz, P3, T4 and 02. In Table 2, the estimates for the calculations of the habituation course are given for $\mathrm{Cz}$ and T4. Figure 3A \& 3C show the averaged ERPs of the two PVAQ groups for $\mathrm{Cz}$ and $\mathrm{P} 4$ in which 25 trials are averaged and time effects cannot be visualized. The right side of Figure 3 (Figures 3B \& 3D), displays the computed (dis)habituation time course for low and high PVAQ scores for the 480 to $600 \mathrm{~ms}$ range for the 25 stimuli, based on the model estimates for the linear and quadratic time functions
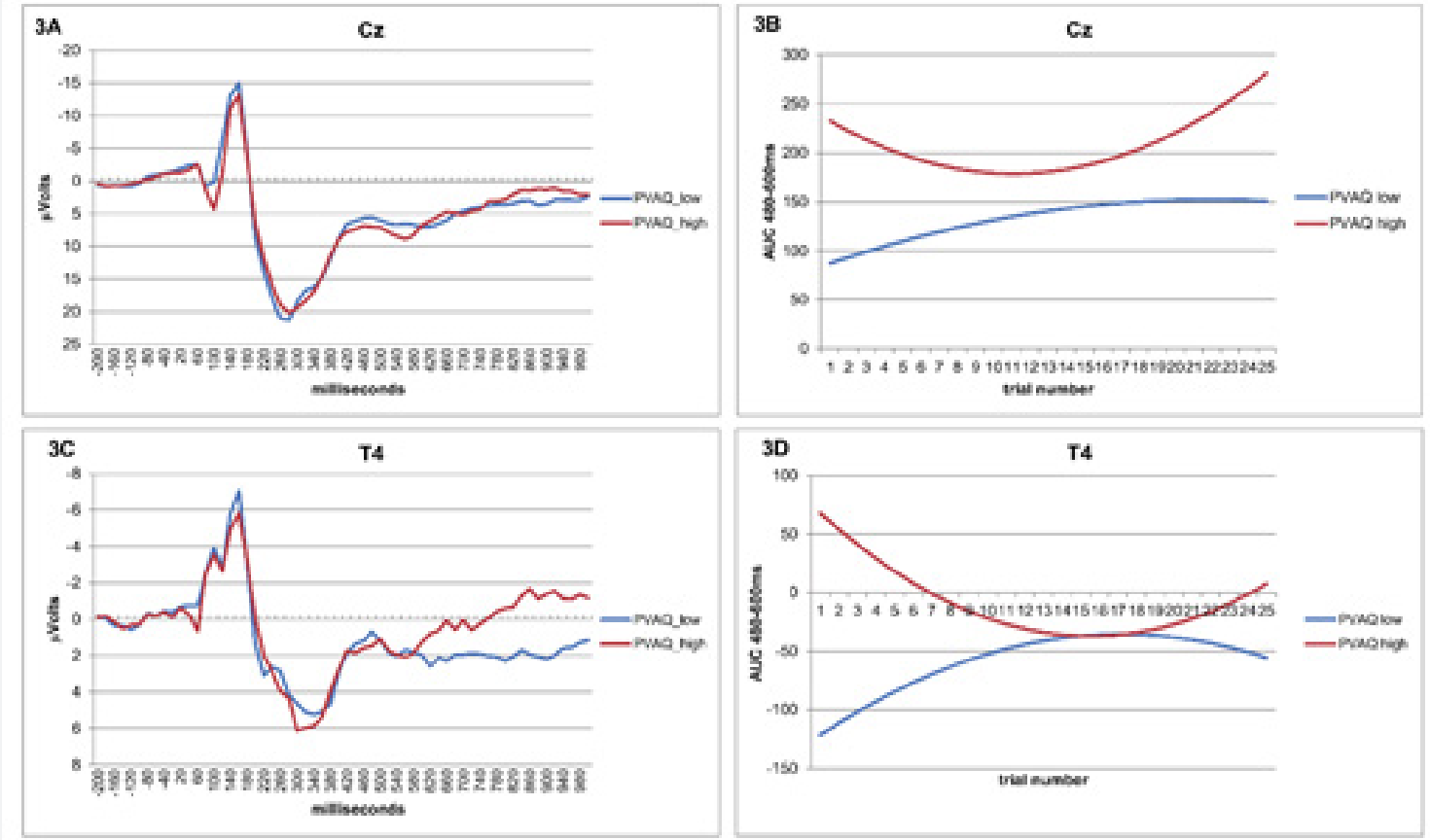

Figure 3A-3D: The influence of PVAQ on habituation for $C z$ an $T 4$ at 480 to $600 \mathrm{~ms}$. On the left the grand averaged ERPs of $C z(3 A)$ and T4 (3C) for both PVAQ groups are depicted. On the right, the AUC time courses over 25 stimuli (3B \& 3D) for both PVAQ groups are visualized. These time courses are based on model estimates summarized in Table 2.

\section{Discussion}

This study investigated two related questions concerning pain hypervigilance in pain-free individuals: Is pain hypervigilance associated with cortical processing of painful stimuli, and, if so, does pain hypervigilance moderate the level of habituation to painful stimuli? With regard to the first question, the answer appeared to be affirmative. A main effect for pain hypervigilance was demonstrated from 440 to $580 \mathrm{~ms}$ post stimulus. A high PVAQ score resulted in a larger (more positive) AUC, which may be interpreted as a stronger processing of the stimulus. The range in which the association was found, however, did not correspond with the a priori expected regions (N140, P300), which are thought to em- bed pain-related information. Moreover, the PVAQ main effect was independent of pain-related variables such as habituation (time course) processes as well as pain and sensation threshold.

Secondly, our data indicate that pain hypervigilance interacts with habituation in the region from 480 to $600 \mathrm{~ms}$. In addition, post-hoc analyses of the sum of these ERFIAs demonstrate that the influence of PVAQ on habituation appears to be relatively robust. Figures 3B \& 3D show the difference in the habituation course over the 25 trials between high and low PVAQ for $\mathrm{Cz}$ and T4. In the high PVAQ group, the habituation course is a parabola that opens upward, whereas the parabola opens downward in the low PVAQ group. The interpretation of these opposite habituation 
parabolic curves is as follows: the high PVAQ group shows increasingly positive ERFIAs in the second part of the experiment. This is indicative of a stronger cortical processing of the stimuli. The opposite process, where the parabola opens downward, a relative decrease in AUC size is apparent for the low PVAQ group in the second part of the experiment. All other electrodes show a similar opposite habituation pattern, some being significant, others not. It is important to understand the difference between the averaged ERPs (Figure 3A \& 3C) and the habituation curves. The averaged ERPs indicate an overall (uncorrected) difference between groups, however, optical differences need to be interpreted with caution. First, a 'traditional' averaged ERP does not display information on Table 2: Model estimates for the area under the curve from $480-600 \mathrm{~ms}$ at $\mathrm{Cz}$ and $\mathrm{T} 4$.

\begin{tabular}{|c|c|c|c|c|}
\hline EEG Electrode & Variable & $\boldsymbol{\beta}$ & $95 \% \mathrm{CI}$ & P-value \\
\hline \multirow{6}{*}{$\mathrm{Cz}$} & Intercept & 81.10 & $-76.42-238.62$ & 0.307 \\
\hline & Trial number & 6.53 & $-6.34-19.40$ & 0.319 \\
\hline & Trial number $_{\text {quadratic }}$ & -0.14 & $-0.62-0.32$ & 0.534 \\
\hline & PVAQ median split & 162.71 & $44.67-280.75$ & $0.007^{*}$ \\
\hline & PVAQ*trial number & -18.25 & $-36.48-0.02$ & $0.05^{*}$ \\
\hline & PVAQ*Trial $_{\text {quadratic }}$ & 0.68 & $0.01-1.34$ & $0.046^{*}$ \\
\hline \multirow{6}{*}{$\mathrm{T} 4$} & Intercept & -132.43 & $-260.10-4.79$ & 0.042 \\
\hline & Trial number & 11.30 & $1.06-21.56$ & 0.031 \\
\hline & Trial number $_{\text {quadratic }}$ & -0.33 & $-0.70-0.04$ & 0.079 \\
\hline & PVAQ median split & 215.41 & $104.91-325.90$ & $<0.001^{*}$ \\
\hline & PVAQ*trial number & -26.77 & $-41.48-12.06$ & $<0.001^{*}$ \\
\hline & PVAQ*Trial $_{\text {quadratic }}$ & 0.83 & $0.30-1.35$ & $0.002^{*}$ \\
\hline
\end{tabular}

PVAQ: Pain Vigilance Awareness Questionnaire: Cl, Confidence Interval. the variance. Second, trial effects inherently disappear through averaging. A typical example can be seen on the T4 electrode of the averaged ERP (Figure 3C). From 480 to $600 \mathrm{~ms}$, a difference between the high and low PVAQ group is practically invisible. The habituation course in this range, however, differs highly significantly between the groups (Table 2). In sum, an averaged ERP only reveals part of the whole story. It is of great importance to analyze the ERP with a multilevel technique in which single trials are nested within subjects. In this way, dynamic time changes in the brain can be unraveled, resulting in a more in-depth understanding of the cortical processing of painful stimuli.

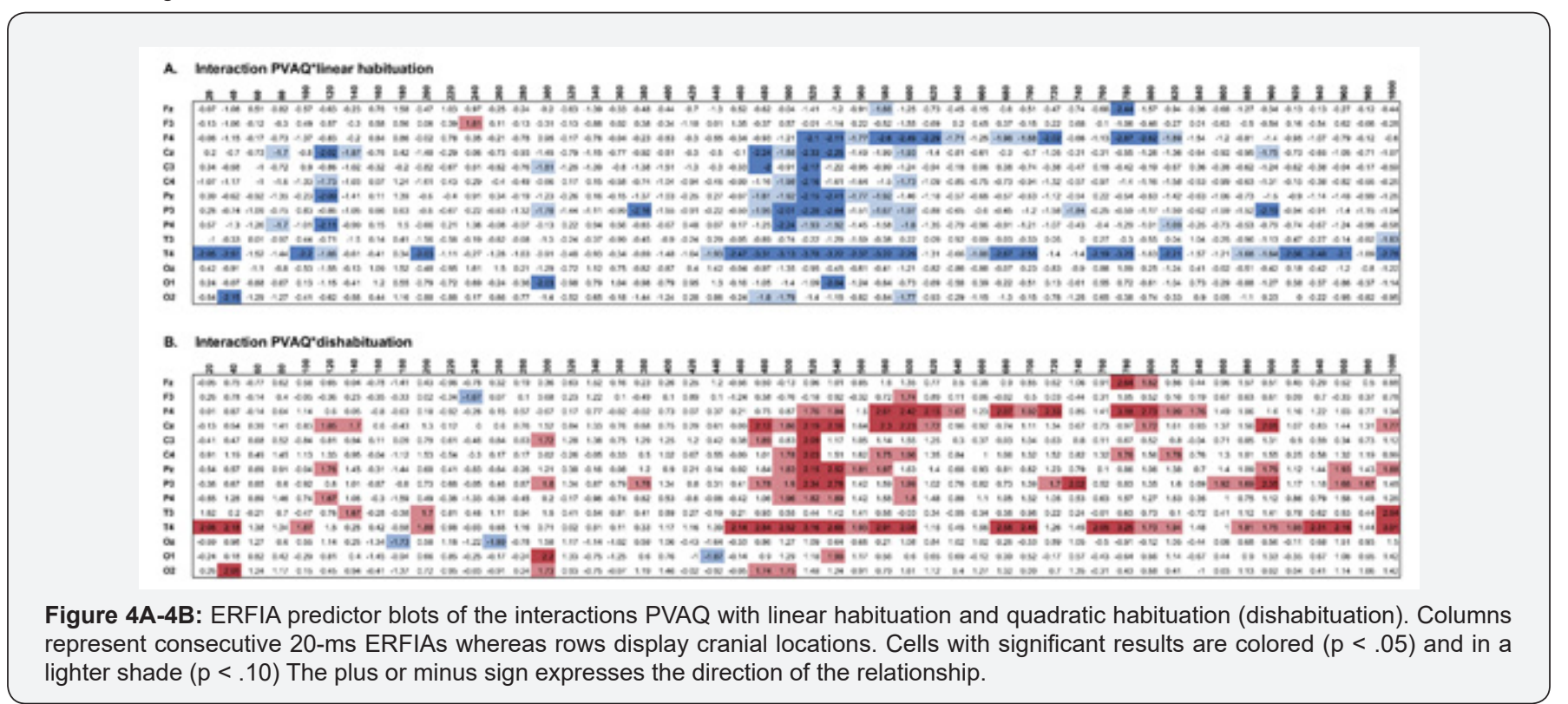

Although an effect of pain hypervigilance on cortical processing is demonstrated, this association could not be 'translated' to an effect on NRS pain report (Figure 1). This discrepancy in findings may be related to several factors. First, it is known that the so-called threat value of a stimulus influences the pain experience [38-41]. In our study, the pain experiences were provoked with- in an experimental setting. In such a setting the participant has an intrinsic control on the experiment by being able to stop the experiment at any time. Therefore, the threat value in this experiment may be perceived as relatively low. Second, Crombez [42] and colleagues questioned whether hypervigilance directly amplifies the experience of pain [42]. The idea is that hypervigilance 
rather intensifies escape or avoidance tendencies, and that amplification of pain can be a result of repeated failure to distract from pain $[42,43]$. The NRS scores in this experiment only measures the intensity aspect of pain, although it is conceivable that hypervigilance may influence other behavioral and affective aspects of pain. Third, hypervigilance may act as a risk-factor in healthy, pain-free individuals in whom enhanced pain processing in the EEG is already manifest, but the actual reporting of pain is still unaffected [9]. Certain conditions or triggers such as acute or chronic pain or an emotional state such as anxiety and depression, need to be present before hypervigilance as a risk factor may result in higher perceived pain intensity [44-46]. In accordance with this line of thought, a study by Lautenbacher demonstrated that hypervigilance was a predictive factor, for postoperative pain, accounting for $17 \%$ of the variance, in a preoperatively pain-free study population [10].

In the present study, which included pain-free subjects, a relationship of PVAQ with pain report was not found. However, in chronic pain patients, pain hypervigilance is associated with a more pronounced pain experience $[3,5,6,47]$. In addition, previous ERP research provides evidence that several chronic pain populations display a decreased habituation compared to painfree controls $[30,37,48]$. Taken together, a mechanistic process in the development of (chronic) pain may unravel as follows: starting with a high level of pain hypervigilance, antinociceptive processes such as habituation may be reduced. After being exposed to repeated painful events, a hypervigilant subject may be more vulnerable to develop chronic pain. It would therefore be instructive to attempt to replicate this study in a sample of chronic pain subjects.

\section{Limitations}

The overall PVAQ score of our study population (mean 28) was significantly lower than the reported mean in the Dutch validation study in pain free individuals (mean 34.4) but did not differ from a healthy presurgical group with a mean PVAQ score of 32 $[8,10]$. The high PVAQ group of our study had a mean score of 39. 5 (SD 7.4), which is comparable to reported means in chronic pain populations such as fibromyalgia (mean 40 SD 12.1) [2]. Therefore, we consider the contrast between the PVAQ groups in our study to be adequate.

Pain hypervigilance was measured with the PVAQ questionnaire, which investigates an explicit form of hypervigilance. Hypervigilance is thought to be a partly automatic process, which is unintentional, uncontrollable and unconscious [42]. We did not account for the implicit form of hypervigilance which can be measured with attentional experiments such as the dot-probe task The results only show that PVAQ has an effect on the habitation of 25 painful electrical stimuli. However, we do not know if this effect is restricted to painful stimuli or that it is a general effect on habituation of various stimuli. A next study should incorporate a non-painful condition to evaluate, whether the PVAQ effect is specific for painful stimuli. Due to the explorative nature of this study, the number of tests was large. Strict corrections, however, may be too conservative and may discard relevant findings. In the future, the number of tests can be decreased by testing a specific latency range based on previous findings. For PVAQ we suggest the range of 480 to $600 \mathrm{~ms}$. As a first attempt, a post-hoc analysis was performed for the region of interest in which PVAQ showed a significant effect. In this experiment, only 14 EEG electrodes were used. Consequently, spatial information could not be assessed. A combined EEG study with fMRI would be of interest to investigate both temporal and spatial information regarding the influence of pain hypervigilance on habituation.

\section{Conclusion}

This study investigated the influence of pain hypervigilance, measured with the PVAQ questionnaire, on cortical processing of painful stimuli, as well as its habituation. A main effect of pain hypervigilance was demonstrated from 440 to $580 \mathrm{~ms}$ post stimulus. In addition, pain hypervigilance interacted with the habituation course in the region from 480 to $600 \mathrm{~ms}$. In conclusion, pain hypervigilance impacts cortical processing of pain and is associated with dishabituation to painful stimuli in healthy subjects, suggesting that hypervigilance may modulate the pain experience through altered cortical habituation.

\section{Acknowledgement}

We are grateful to Marga Schnitzeler, for the recruitment of participants, performing the EEG measurements and data management and to Dr. Wolfgang Viechtbauer for statistical advice, both from the Department of Psychiatry and Neuropsychology, Maastricht University Medical Centre.

\section{References}

1. Chapman CR (1978) Pain: The Perception of Noxious Events. In: Sternbach R (Ed.), In the Psychology of Pain Raven Press, New York, USA.

2. Roelofs J, Peters ML, McCracken L, Vlaeyen JWS (2003) The pain vigilance and awareness questionnaire (PVAQ): Further psychometric evaluation in fibromyalgia and other chronic pain syndromes. Pain 101(3): 299-306.

3. McCracken LM (1997) "Attention" to pain in persons with chronic pain: A behavioral approach. Behav Ther 28(2): 271-284.

4. Eccleston C, Crombez G (1999) Pain demands attention: a cognitive-affective model of the interruptive function of pain. Psychol Bull 125(3): 356-366.

5. Crombez G, Eccleston C, Baeyens F, Van Houdenhove B, Van Den Broeck A (1999) Attention to chronic pain is dependent upon pain-related fear. J Psychosom Res 47(5): 403-410.

6. Crombez G, Eccleston C, Van den Broeck A, Goubert L, Van Houdenhove B (2004) Hypervigilance to pain in fibromyalgia: the mediating role of pain intensity and catastrophic thinking about pain. Clin J Pain 20(2): 98-102.

7. McWilliams LA, Asmundson GJG (2001) Assessing individual differences in attention to pain: Psychometric properties of the Pain Vigilance and Awareness Questionnaire modified for a non-clinical pain sample. Pers Individ Dif 31(2): 239-246.

8. Roelofs J, Peters ML, Muris P, Vlaeyen JWS (2002) Dutch version of the Pain Vigilance and Awareness Questionnaire: Validity and reliability in a pain-free population. Behav Res Ther 40(9): 1081-1090. 
9. Rollman GB (2009) Perspectives on hypervigilance. Pain 141(3): 183184.

10. Lautenbacher S, Huber C, Kunz M, Parthum A, Weber PG, et al. (2009) Hypervigilance as predictor of postoperative acute pain: its predictive potency compared with experimental pain sensitivity, cortisol reactivity, and affective state. Clin J Pain 25(2): 92-100.

11. Lautenbacher S, Huber C, Schöfer D, Kunz M, Parthum A, et al. (2010) Attentional and emotional mechanisms related to pain as predictors of chronic postoperative pain: A comparison with other psychological and physiological predictors. Pain 151(3): 722-731.

12. Slade GD, Diatchenko L, Bhalang K, Sigurdsson A, Fillingim RB, et al. (2007) Influence of psychological factors on risk of temporomandibular disorders. J Dent Res 86(11): 1120-1125.

13. Latremoliere A, Woolf CJ (2009) Central Sensitization: A Generator of Pain Hypersensitivity by Central Neural Plasticity. J Pain 10(9): 895926.

14. Herbert MS, Goodin BR, Pero ST, Schmidt JK, Sotolongo A, et al. (2013) Pain Hypervigilance is Associated with Greater Clinical Pain Severity and Enhanced Experimental Pain Sensitivity Among Adults with Symptomatic Knee Osteoarthritis. Ann Behav Med 48(1): 50-60.

15. Rennefeld C, Wiech K, Schoell ED, Lorenz J, Bingel U (2010) Habituation to pain: further support for a central component. Pain 148(3): 503-508.

16. Thompson RF, Spencer WA (1966) Habituation: a model phenomenon for the study of neuronal substrates of behavior. Psychol Rev 73(1): 16-43.

17. Rankin CH, Abrams T, Barry RJ, Bhatnagar S, Clayton DF, et al. (2009] Habituation revisited: an updated and revised description of the behavioral characteristics of habituation. Neurobiol Learn Mem 92(2): 135-138.

18. Bingel U, Schoell E, Herken W, Büchel C, May A (2007) Habituation to painful stimulation involves the antinociceptive system. Pain 131(1-2): 21-30.

19. Bingel U, Schoell E, Herken W, Büchel C, May A (2008) Habituation to painful stimulation involves the antinociceptive system- a 1-year follow-up of 10 participant. Pain 140(2): 393-394.

20. Melzack R (1999) From the gate to the neuromatrix. Pain Suppl 6: S121-S126.

21. Luck SJ (2005) Ten simple rules for designing ERP experiments. In Event-related potentials: a methods handbook (Handy TC, ed.), MIT Press, pp. 17-23.

22. Becker DE, Yingling CD, Fein G (1993) Identification of pain, intensity and P300 components in the pain evoked potential. Electroencephalogr Clin Neurophysiol 88(4): 290-301.

23. Bromm B, Meier W (1984) The intracutaneous stimulus: a new pain model for algesimetric studies. Methods Find Exp Clin Pharmacol 6(7): 405-410.

24. Bromm B, Lorenz J (1998) Neurophysiological evaluation of pain. Electroencephalogr Clin Neurophysiol 107(4): 227-253.

25. Iannetti GD, Zambreanu L, Cruccu G, Tracey I (2005) Operculoinsular cortex encodes pain intensity at the earliest stages of cortical processing as indicated by amplitude of laser-evoked potentials in humans. Neuroscience 131(1): 199-208.

26. Miltner W, Johnson R, Braun C, Larbig W (1989) Somatosensory event-related potentials to painful and non-painful stimuli: effects of attention. Pain 38(3): 303-312.

27. Le Pera D, Valeriani M, Niddam D, Chen AC, Arendt-Nielsen L (2002) Contact heat evoked potentials to painful and non-painful stimuli: effect of attention towards stimulus properties. Brain Topogr 15(2): 115-123.

28. Milne RJ, Kay NE, Irwin RJ (1991) Habituation to repeated painful and non-painful cutaneous stimuli: a quantitative psychophysical study. Exp Brain Res 87(2): 438-444.

29. Valeriani M, De Tommaso M, Restuccia D, Le Pera D, Guido M, et al (2003) Reduced habituation to experimental pain in migraine patients: a CO(2) laser evoked potential study. Pain 105(1-2): 57-64.

30. De Tommaso M, Federici A, Santostasi R, Calabrese R, Vecchio E, et al. (2011) Laser-evoked potentials habituation in fibromyalgia. J Pain 12(1): 116-124.

31. Vossen CJ, Vossen HGM, Marcus MAE, Van Os J, Lousberg R (2013) Introducing the event related fixed interval area (ERFIA) multilevel technique: A method to analyze the complete epoch of event-related potentials at single trial level. PLoS One 8(11).

32. Aaronson NK, Muller M, Cohen PDA, Essink Bot ML, Fekkes M, et al. (1998) Translation, Validation, and Norming of the Dutch Language Version of the SF-36 Health Survey in Community and Chronic Disease Populations. J Clin Epidemiol 51(11): 1055-1068.

33. Daut RL, Cleeland CS, Flanery RC (1983) Development of the Wisconsin Brief Pain Questionnaire to assess pain in cancer and other diseases. Pain 17(2): 197-210.

34. Shahid A, Wilkinson K, Marcu S, Shapiro CM (2011) Brief Pain Inventory (BPI) In STOP, THAT and One Hundred Other Sleep Scales. Springer, New York, USA, pp. 81-88.

35. Bromm B, Lorenz J (1998) Neurophysiological evaluation of pain. Electroencephalogr Clin Neurophysiol 107(4): 227-253.

36. Klem GH, Lüders HO, Jasper HH, Elger C (1999) The ten-twenty electrode system of the International Federation. The International Federation of Clinical Neurophysiology. Electroencephalogr Clin Neurophysiol 52: 3-6.

37. Vossen CJ, Vossen HGM, Joosten EA, Van Os J, Lousberg R (2015) Does habituation differ in chronic low back pain subjects compared to painfree controls? A cross-sectional pain rating ERP study reanalyzed with the ERFIA multilevel method. Medicine (Baltimore) 94(19): e865.

38. Aldrich S, Eccleston C, Crombez G (2000) Worrying about chronic pain: Vigilance to threat and misdirected problem solving. Behav Res Ther 38(5): 457-470.

39. Crombez G, Eccleston C, Baeyens F, Eelen P (1998) When somatic information threatens, catastrophic thinking enhances attentional interference. Pain 75(2-3): 187-198.

40. Van Damme S, Crombez G, Eccleston C, Koster EHW (2006) Hypervigilance to Learned Pain Signals: A Componential Analysis. J Pain 7(5): 346-357.

41. Moore DJ, Keogh E, Eccleston C (2013) The effect of threat on attentional interruption by pain. Pain 154(1): 82-88.

42. Crombez G, Van Damme S, Eccleston C (2005) Hypervigilance to pain: An experimental and clinical analysis. Pain 116(1-2): 4-7.

43. Crombez G, Vervaet L, Baeyens F, Lysens R, Eelen P (1996) Do pain expectancies cause pain in chronic low back patients? A clinical investigation. Behav Res Ther 34(11-12): 919-925.

44. Vlaeyen JWS, Linton SJ (2000) Fear-avoidance and its consequences in chronic musculoskeletal pain: A state of the art. Pain 85(3): 317-332.

45. Janssen SA (2002) Negative affect and sensitization to pain. Scand J Psychol 43(2): 131-137.

46. Huber C, Kunz M, Artelt C, Lautenbacher S (2010) Attentional and emotional mechanisms of pain processing and their related factors: A structural equations approach. Pain Res Manag 15(4): 229-237. 
47. Eccleston C, Crombez G, Aldrich S, Stannard C (1997) Attention and somatic awareness in chronic pain. Pain 72(1-2): 209-215.

This work is licensed under Creative Commons Attribution 4.0 License

DOI: 10.19080/JAICM.2019.08.555738
48. De Tommaso M, Libro G, Guido M, Losito L, Lamberti P, et al. (2005) Habituation of single $\mathrm{CO}_{2}$ laser-evoked responses during interictal phase of migraine. J Headache Pain 6(4): 195-198.

\section{Your next submission with Juniper Publishers} will reach you the below assets

- Quality Editorial service

- Swift Peer Review

- Reprints availability

- E-prints Service

- Manuscript Podcast for convenient understanding

- Global attainment for your research

- Manuscript accessibility in different formats

(Pdf, E-pub, Full Text, Audio)

- Unceasing customer service

Track the below URL for one-step submission

https://juniperpublishers.com/online-submission.php 